\title{
APLICAÇÃO DE SOFTWARE DE ANÁLISE DE TENSÃO RESIDUAL EM AMOSTRAS METÁLICAS LAMINADAS*
}

\author{
Ariel José Assayag do Lago ${ }^{1}$ \\ Beatriz Sampaio Torres ${ }^{1}$ \\ Géssica Seara Faria ${ }^{2}$ \\ Juciane Maria Alves ${ }^{3}$ \\ Talita Gama de Sousa ${ }^{3}$ \\ Hugo Serrão Ribeiro Borges ${ }^{4}$ \\ Rebeca Pinheiro dos Reis ${ }^{4}$ \\ Lucas Alexandre Golçalves Lima \\ Luiz Paulo Mendonça Brandão ${ }^{5}$
}

\section{Resumo}

O presente trabalho teve por finalidade analisar as tensões residuais, através da técnica de difração de raios- $X$, em amostras de cobre submetidas à laminação em temperatura ambiente. Foram obtidos e comparados os resultados de um software comercial com um software projetado pelos autores. O comportamento das tensões principais das amostras laminadas foi comparado com o resultado do material no estado recozido. Uma tendência de aumento de tração trativa no interior da amostra foi identificada. Os resultados obtidos seguiram um comportamento dentro do previsto pela bibliografia e os dois softwares utilizados acusaram valores similares, ratificando a aplicabilidade do método de difração assim como a funcionalidade do software desenvolvido.

Palavras-chave: DRX; Tensão Residual; Cobre.

\section{APLICATION OF RESIDUAL STRESS ANALYSIS SOFTWARE ON COLD ROLLED METALLIC SPECIMENS}

\section{Abstract}

This paper intends to analyze residual stress on copper specimens processed by cold rolling. The values of main stress were acquired by X-ray diffraction with the Psi geometry method. The results were obtained utilizing commercial software and software designed by the authors. The behavior of main stress values of cold rolled specimens was compared with the behavior of annealed copper. A increase tendency of the residual stress on the internal layers of the specimens with the reduction by lamination was observed. The results obtained adopted a pattern predicted by the literature and values from both the commercial and the undergraduate software were close to each other, thus emphasizing the applicability of the diffraction method and also the functionality of the software.

Keywords: XRD; Residual Stress; Copper.

1 Aluno de Graduação - Engenharia de Materiais, Seção de Engenharia Mecânica e de Materiais, Instituto Militar de Engenharia - Rio de Janeiro, RJ, Brasil.

2 Engenheira Metalúrgica - Mestranda em Engenharia de Materiais, Seção de Engenharia Mecânica e de Materiais - Instituto Militar de Engenharia, Rio de Janeiro, RJ, Brasil.

3 Química, M.Sc., Doutoranda em Ciência dos Materiais no Instituto Militar de Engenharia - IME, Rio de Janeiro, RJ, Brasil.

4 Aluno de graduação - Curso Básico, Seção de Ensino Básico, Instituto Militar de Engenharia, Rio de Janeiro, RJ, Brasil.

5 Engenheiro Metalúrgico, D.Sc., Professor Associado, Seção de Engenharia Mecânica e de Materiais, Instituto Militar de Engenharia - IME, Rio de Janeiro, RJ, Brasil. 


\section{INTRODUÇÃO}

Uma das utilidades da prática da difração de raios-X (DRX) consiste na análise das tensões residuais presentes na amostra. Essas se caracterizam por serem tensões que permanecem no material mesmo sem a aplicação de forças externas e/ou mudança de temperatura. Elas podem ser de natureza trativa ou compressiva e são provenientes de transformações de fase, processos de conformação, tratamentos mecânicos superficiais, soldagem, dentre outros[1].

Se a distribuição dessas tensões for de caráter uniforme ao longo de grandes distâncias na rede cristalina serão chamadas de macrotensões e provocam deslocamento na posição do pico de difração, estas estão relacionadas principalmente a processos de conformação mecânica. Se, no entanto, em um mesmo grão houver regiões com diferentes espaçamentos interplanares, estas tensões serão denominadas microtensões residuais, oriundas de deformações plásticas. Estas microtensões são responsáveis por um alargamento do pico de difração, sendo ele formado pelo somatório de várias linhas de difração. Estes efeitos podem se sobrepor em materiais cristalinos deformados plasticamente[2].

Sendo a análise realizada através da difração de raios- $X$, deve-se considerar o caráter superficial da mesma, o qual torna necessária a remoção de tensões superficiais, como também, a remoção de material caso seja requerida averiguação de tensão no interior da amostra. A análise se baseia na medição não diretamente das tensões, mas sim da diferença entre as distâncias interplanares causada pelas deformações geradas pelos esforços mecânicos, térmicos, inserção de nova fase ou cristaloquímicos. A relação dessas tensões geradas com as deformações causadas pela variação de distância interplanar é possível através da Lei de Hooke (Equação 1) onde $E$ é o módulo de elasticidade do material. Além disso, a correlação entre as deformações nos diferentes eixos pode ser feita através do coeficiente de Poisson, onde este é obtido através da Equação 2.

$$
\begin{aligned}
\sigma & =E . \epsilon \\
v=-\frac{\epsilon_{x}}{\epsilon_{z}} & =-\frac{\epsilon_{y}}{\epsilon_{z}}
\end{aligned}
$$

O método utilizado para análise de tensão residual foi o Método Geometria Psi, o qual consiste na aferição da variação das distâncias interplanares em diferentes rotações do ângulo $\phi$ (rotação da amostra) e na correlação dessas variações com $\operatorname{sen}^{2} \psi$ (inclinação da amostra). Essa relação pode ser representada, após desenvolvimento matemático com base na teoria da Elasticidade, através da Equação 3:-

$$
\epsilon_{\psi \phi}=\frac{1+v}{E} \sigma_{\phi} \operatorname{sen}^{2} \Psi+\epsilon_{1}
$$

Onde $\epsilon_{\psi \phi}$ representa a deformação da rede cristalina e $\sigma_{\phi}$ representa a tensão residual existente na rede-[3]. 


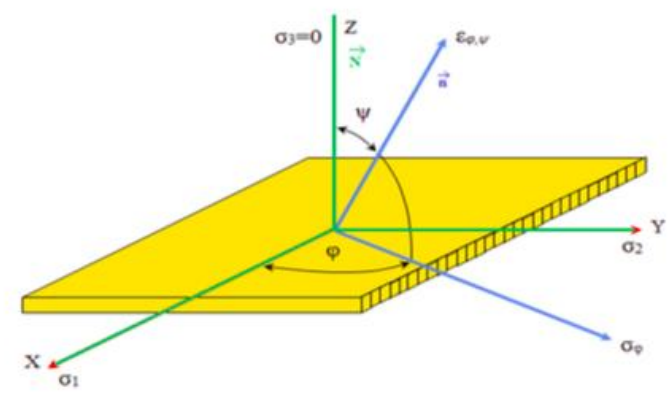

Figura 1: Geometria $\boldsymbol{\psi}$ na superfície da amostra(ALVES, J.M.).

À entidade matemática representada pelas tensões normais e cisalhantes atuantes em um corpo dá-se o nome de tensor, o qual é apresentado na forma de uma matriz $3 \times 3$ com três componentes normais e seis cisalhantes. A ordem da matriz indica o número de dimensões do estado de tensões analisado. Na superfície da amostra, não há atuação de esforços normais à área medida. Portanto, a componente normal de tensão é nula e o estado de tensão é plano. Então, busca-se, por meio da difração de raios- $X$, averiguar as tensões residuais que caracterizam o estado plano de tensão da amostra.

O estudo dessas tensões em um material é portador de grande relevância, visto que estas podem ser responsáveis por consideráveis modificações nas propriedades do mesmo. Esta análise também possui grande importância no estudo da falha dos materiais, pois está diretamente relacionada a mecanismos de aumento ou atenuação da resistência mecânica, propagação de trinca em fadiga e outros processos[4].

Em um processo de laminação- a distribuição de macrotensões assume um perfil característico, ou seja, as camadas superficiais, em contato com os cilindros são deformadas mais intensamente do que o interior. Ao cessar a aplicação de forças, o esforço do material de manter a seção transversal plana leva o exterior do corpo a apresentar tensão residual compressiva e interior a apresentar tensão residual trativa[2].

Entretanto, sobrepõe-se às macrotensões o complexo estado de microtensões advindas das intensas deformações plásticas [5]. O método difração não distingue entre os dois tipos de tensão residual. Logo, o que se analisa no experimento não é o verdadeiro estado de macrotensões. Vale ressaltar que para a análise de tensões residuais deve-se ter uma atenção especial ao preparo das amostras. A preparação metalográfica deve ser realizada de modo a remover as tensões superficiais do material, resultantes da etapa de lixamento, utilizando-se para isto polimento químico ou eletrolítico.

\section{MATERIAIS E MÉTODOS}

O material em estudo consistiu em uma chapa de cobre comercialmente puro (Tabela 1), recozida a $600^{\circ} \mathrm{C}$ por 1 hora com taxa de aquecimento de $10^{\circ} \mathrm{C} /$ minuto e resfriamento ao forno. Realizou-se laminação, à temperatura ambiente, com percentuais de redução de 30 e $50 \%$. A preparação metalográfica foi realizada utilizando-se lixas de 400, 600, 1200 e 2000 mesh, polimento mecânico com pasta de diamante de $1 \mu \mathrm{m}$ e posteriormente realizou-se polimento químico com solução composta por ácido acético, nítrico e fosfórico (proporção 1 parte $+1 / 4: 1$ parte : 2 partes $+3 / 4)$ respectivamente. $O$ tempo de imersão das amostras, durante 0 
polimento químico, foi entre 30 e 40 segundos e a temperatura da solução foi próxima a $60^{\circ} \mathrm{C}$. Com o objetivo de avaliar as tensões residuais no interior do material as amostras foram desbastadas à meia espessura.

Para a análise de tensão residual utilizou-se um difratômetro com goniômetro de textura $\theta-\theta$, apresentado na Figura 2. Durante a medição a amostra foi rotacionada a $0^{\circ}, 45^{\circ}$ e $90^{\circ}$ em $\phi$, e inclinada em $\psi$ com angulações de $-65^{\circ}$ a $65^{\circ}$ em nove passos; correspondentes aos ângulos $0^{\circ}, 26,95^{\circ}, 39,86^{\circ}, 51,71^{\circ}, 65,00^{\circ},-26,95^{\circ}$, $39,86^{\circ},-51,71^{\circ}$ e $-65^{\circ}$. Isso resulta em um gráfico $d$ versus $\operatorname{sen}^{2} \psi$ com nove pontos distintos (Figuras 5, 6 e 7).

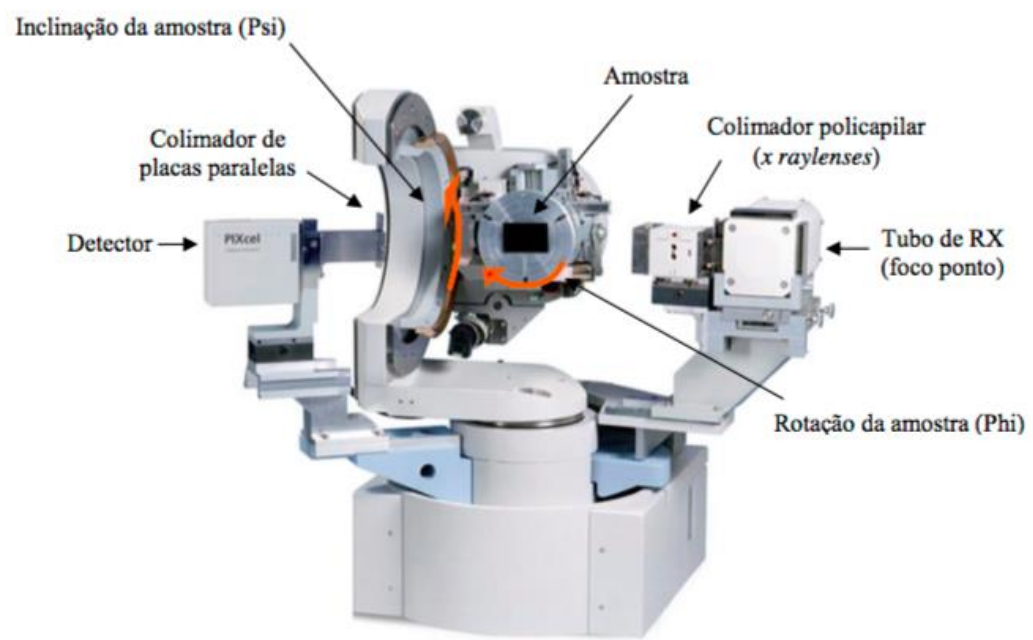

Figura 2: Representação dos ângulos $\phi$ e $\psi$ no difratômetro utilizado. (ALVE, J.M.).

A precisão dessas análises é fundamental. Sendo assim, é necessária a seleção do pico de maior angulação, $2 \theta=110^{\circ}$, o qual tem maior precisão para detectar a presença de deformações por tensão. Associado a isto, utiliza-se a configuração de foco ponto, com a finalidade de reduzir a desfocagem e o ruído de fundo das medidas.

Foram realizados testes previamente ao experimento para que fosse encontrado um time per step ideal, que conciliasse precisão e rapidez. Valores baixos desse parâmetro acarretariam em perda de precisão e aumento de background. Já valores altos, ocasionariam um ensaio demasiadamente demorado, o qual consumiria excessivamente 0 tubo de raios- $X$. As condições das medidas foram: range 6은, tamanho do passo $0,08^{\circ}$, tempo por passo $6 \mathrm{~s}$, abertura das fendas $4^{\circ} \times 4^{\circ}$, tempo aproximado de análise 3 horas e 45 minutos. Utilizou-se um difratômetro da Panalytical com tubo de cobalto, filtro de ferro e detector PIXcel, tensão e corrente do equipamento $40 \mathrm{kV}$ e $45 \mathrm{~mA}$, respectivamente.

O processamento pós-medida por difração foi realizado o software $X$ ' Pert Stress da Panalytical. Foram adicionados ao refinamento da medida uma série de fatores, os quais direcionam a modelagem do perfil de pico experimental. Dentre os fatores que foram considerados, estão:

- Mudança do método de trabalho;

- Inserção dos valores do módulo de elasticidade, do coeficiente de Poisson e de parâmetros de estrutura cristalina para o cobre;

- Dimensão do estado de tensão; 
- Lorentz-Polarisation;

- Correção de desalinhamento do feixe e da amostra;

- Refinamento de rede;

- Ajuste de background;

A definição destes parâmetros é controlada através software de análise comercial pela interface exibida na Figura 3.

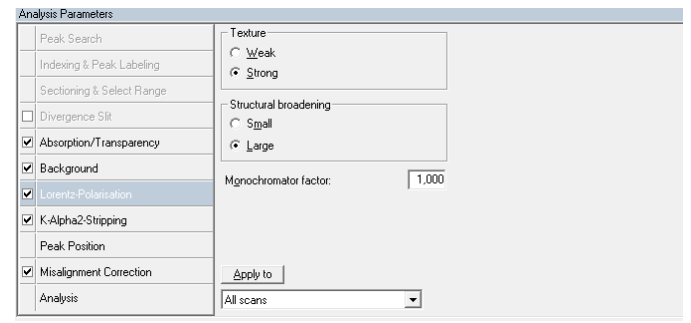

Figura 3: Interface do software comercial utilizado.

Fazendo a modelagem do perfil, pode-se extrair o valor do espaçamento interplanar. Cada pico analisado-fornece um par coordenado de distância interplanar e $\operatorname{sen}^{2} \psi$ (d versus $\operatorname{sen}^{2} \psi$ ) para compor o gráfico do Método de Geometria Psi. Na dispersão de pontos plotada, o software realiza uma regressão linear e, relacionando a equação obtida com a Equação 1, pode-se extrair os valores de tensão desejados.

Os parâmetros de modelagem do software devem ser alterados para que os pontos da dispersão estejam o mais próximo possível da distribuição linear. O desvio deste comportamento acarreta em aumento no erro experimental. Este erro associado ao método utilizado pode ser exposto em termos do desvio padrão dos valores de tensão calculados.

Os dados do experimento também foram analisados por meio do software SATR$\mathrm{DRX}$, desenvolvido pelos autores. O funcionamento do programa tem por base os fundamentos do estudo de tensão residual pelo método da geometria $\psi$.

A partir desta técnica, os resultados obtidos foram analisados e comparados entre si e com a literatura.

\section{RESULTADOS E DISCUSSÃO}

O perfil de pico $2 \theta=110^{\circ}$ da amostra recozida sem deformação plástica pode ser observado na Figura 4. Podem ser observados o pico teórico, em azul, e o pico experimental, em vermelho, levando em consideração os parâmetros de modelagem- 


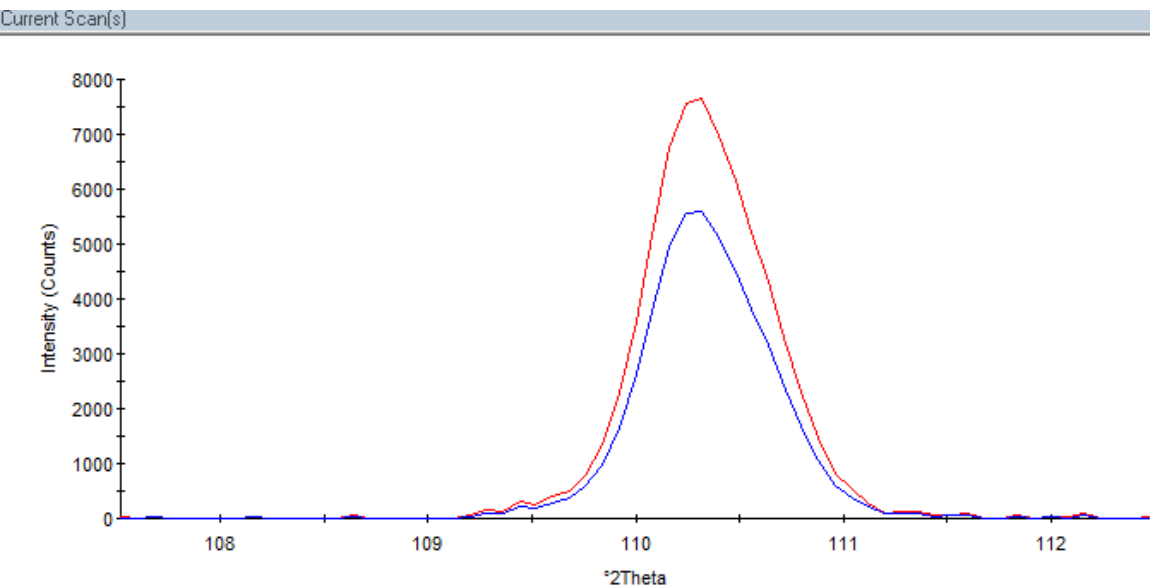

Figura 4: Pico de difração no na interface do software de análise.

Os gráficos de deformação versus $\operatorname{sen}^{2} \psi$ obtidos a partir das amostras analisadas são apresentados nas Figuras 5, 6 e 7.

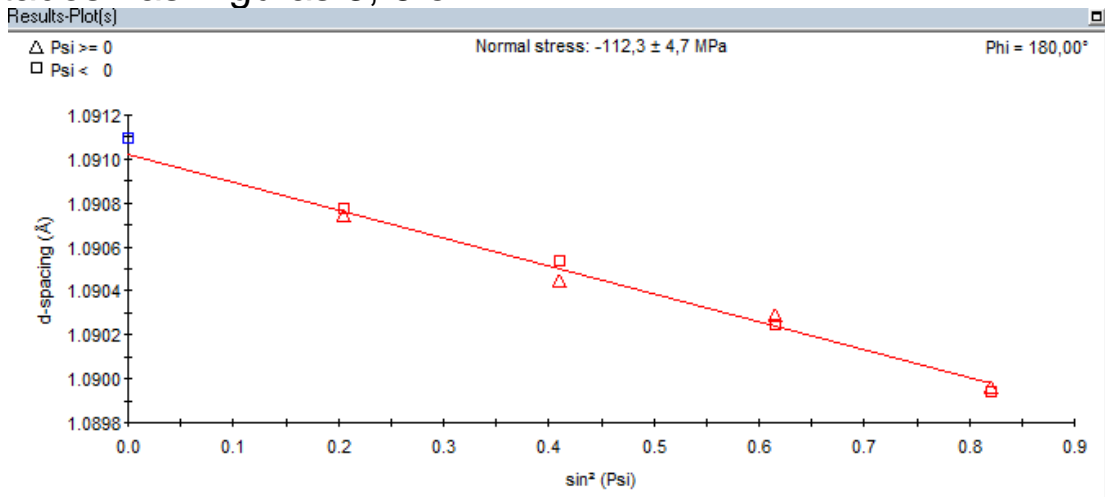

Figura 5: Regressão linear da amostra recozida

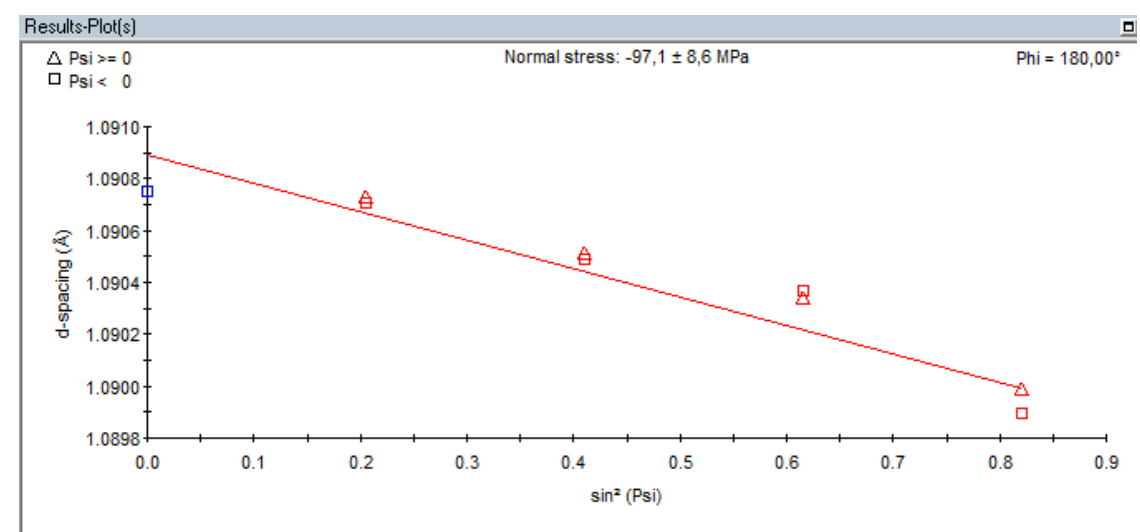

Figura 6: Regressão linear da amostra com 30\% de redução por laminação à temperatura ambiente. 


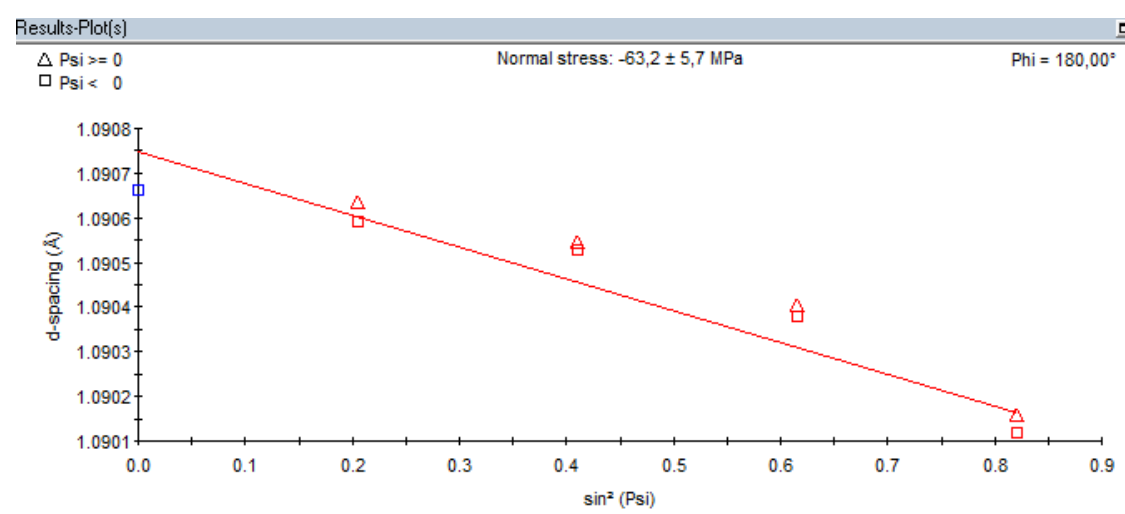

Figura 7: Regressão linear da amostra com $50 \%$ de redução por laminação à temperatura ambiente.

Uma análise qualitativa dos gráficos permite observar um comportamento satisfatório dos pontos descrito por uma reta. Este fato indica que a tensão residual está homogeneamente distribuída nas áreas analisadas.

Por meio da análise numérica da reta obtida para cada amostra, os resultados das tensões principais que descrevem o estado plano de tensões estão expostos na Tabela 1. O coeficiente $\Delta \sigma$ é o desvio padrão oriundo dos valores de tensão medidos para cada ponto.

Tabela 1: Resultados finais de tensões principais.

\begin{tabular}{c|c|c|c}
\hline $\begin{array}{c}\text { Redução } \\
\text { Laminação }\end{array}$ & $\sigma_{11}(\mathrm{MPa})$ & $\sigma_{22}(\mathrm{MPa})$ & $\Delta \sigma(\mathrm{MPa})$ \\
\hline CR & $-112,3$ & $-119,8$ & 4,71 \\
$30 \%$ & $-97,1$ & -98 & 8,63 \\
$50 \%$ & $-63,2$ & $-83,1$ & 5,71 \\
\hline
\end{tabular}

A tendência dos resultados encontrados pode ser analisada graficamente através da Figura 8.

Figura 8: Tendência dos valores de tensão principal medidos das amostras CR e com reduções de 30 e $50 \%$.

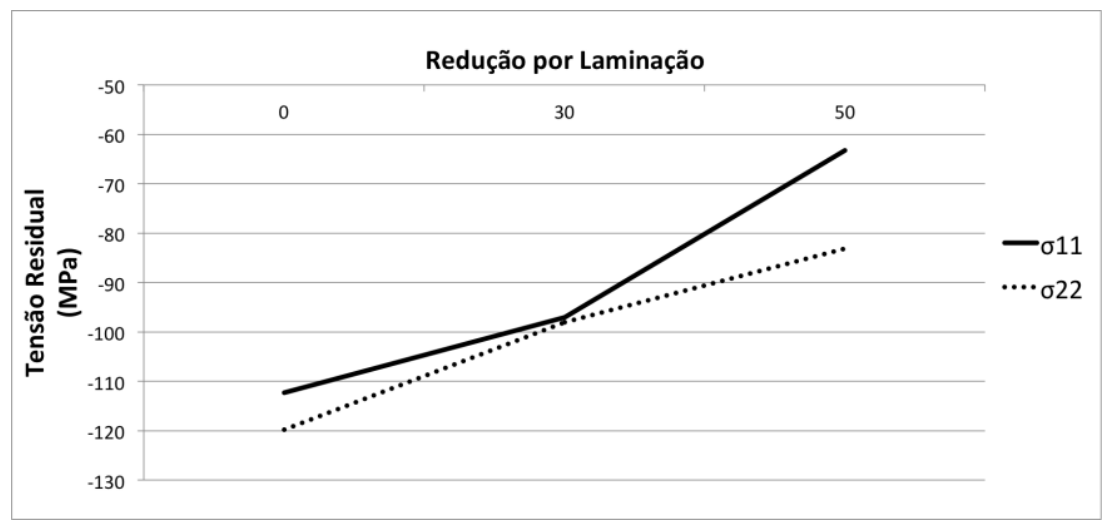


Os resultados da análise de tensão residual utilizando-se o software SATR-DRX são apresentados na Tabela 2.

Tabela 2: Resultados de tensão residual obtidos com o software projetado SATR-DRX.

\begin{tabular}{c|c|c}
\hline $\begin{array}{c}\text { Redução } \\
\text { Laminação }\end{array}$ & $\sigma_{11}(\mathrm{MPa})$ & $\sigma_{22}(\mathrm{MPa})$ \\
\hline CR & $-113,385$ & $-118,383$ \\
$30 \%$ & $-90,723$ & $-91,301$ \\
$50 \%$ & $-63,605$ & $-84,969$ \\
\hline
\end{tabular}

Uma comparação entre os valores obtidos através dos softwares X'Pert Stress e SATR-DRX é apresentada na Figura 9. Pode-se verificar a proximidade entre os resultados obtidos, destacando a coerência da teoria aplicada à análise de tensão residual.

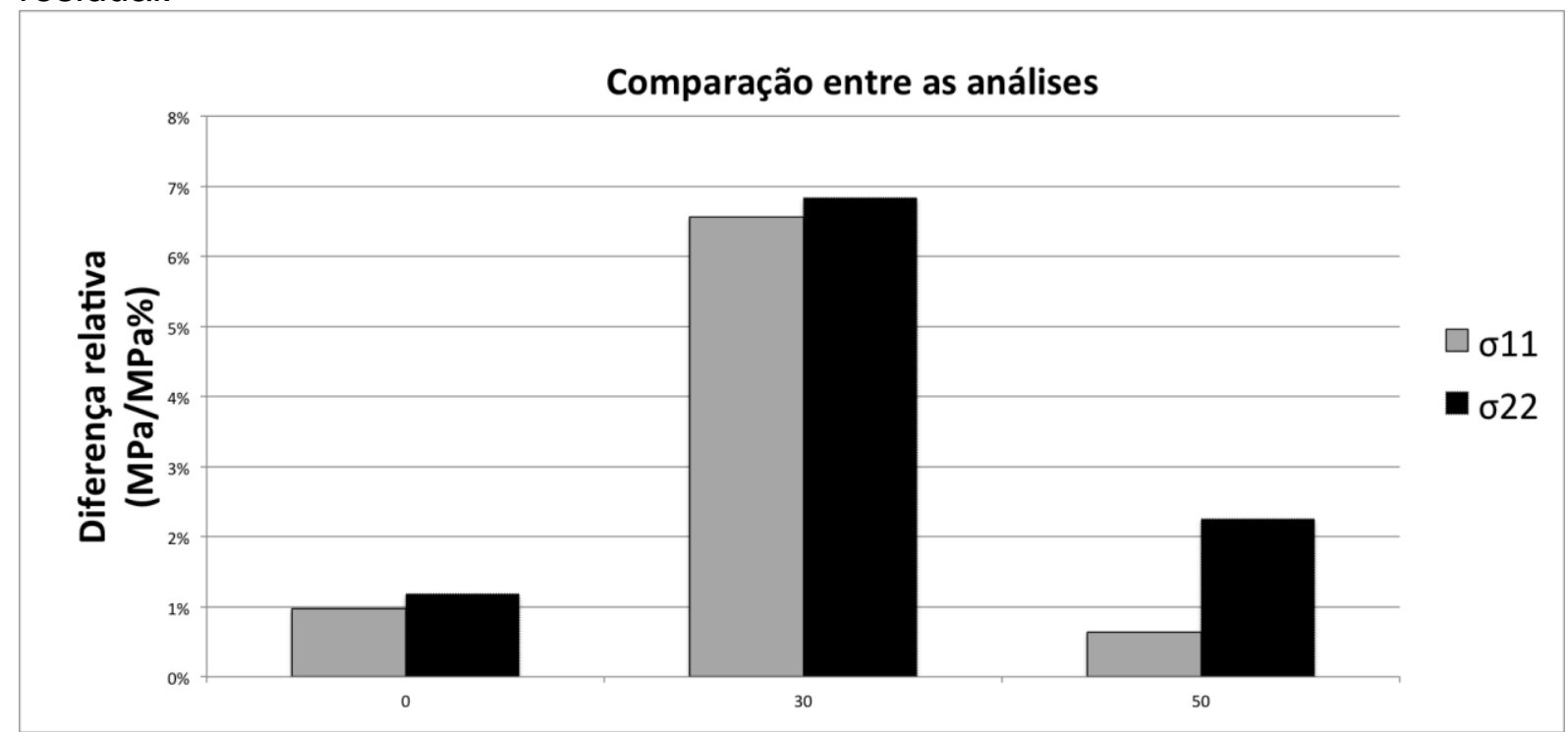

Figura 9: Comparação entre os dados de análise obtidos pelos diferentes softwares.

Por meio da Figura 8 e das Tabelas 1 e 2, pode-se verificar que há uma tendência de aumento da tensão com o aumento da deformação por laminação. Deve-se notar dois pontos importantes para a análise dos resultados. O primeiro consiste na observação de que o processo de recozimento conduz a um alívio nas tensões residuais presentes na estrutura do material. $O$ resultado deste tratamento é um corpo com grãos recristalizados e tensões homogeneamente distribuídas ao longo de seu volume. Portanto, a amostra recozida serve como referência para as análises a serem realizadas. O segundo ponto a ser destacado é a distribuição de macrotensões no processo de laminação discutida anteriormente. Como foi avaliada a tensão residual à meia espessura das amostras, a tensão presente nesta região deve tender à trativa. O que se observa, portanto, para as amostras que sofreram redução por laminação é a superposição das tensões preexistentes na amostra recozida com as tensões trativas oriundas do processo de laminação. Como previsto por esta análise, os valores de tensão principal tendem a assumir valores cada vez menos compressivos com o aumento da deformação, ou seja, estão sendo adicionadas componentes trativas cada vez mais relevantes. Esta tendência é evidente na Figura 9. Não obstante, deve ser ressaltado que quanto maior a deformação por laminação, maior será o acréscimo de estados complexos de 
microtensão na amostra. Este fato dificulta a análise por causa da introdução de heterogeneidades no valor da tensão residual e culmina em um desvio do comportamento linear para a dispersão obtida.

\section{CONCLUSÃO}

A análise de tensão residual por meio da técnica de difração de raios-X permitiu a averiguação do estado de tensões em amostras laminadas. Como o esperado, observou-se a tendência de tensões trativas no interior do material, isto ao se observar valores cada vez menos compressivos com o incremento da redução por laminação. Ou seja, foram adicionadas componentes trativas cada vez mais relevantes ao passo que as amostras foram submetidas à deformação.

Um fator indesejável na análise de tensão residual por difração de raios-X é o tempo elevado de medição, necessário para se concluir um experimento. Porém é um método não destrutivo e permite a análise qualitativa e quantitativa de tensões residuais.

O software projetado SATR-DRX, com desenvolvimento matemático na teoria da Elasticidade, para a análise tensões residuais levou a resultados satisfatórios e concordantes com aqueles obtidos através do X' Pert Stress da Panalytical. As respostas qualitativas e quantitativas foram adequadas e os valores quantitativos seguiram a tendência esperada.

\section{REFERÊNCIAS}

1 J.M.; Análise da Transformação Martensítica e Tensão Residual em um Aço inoxidável 304L, Dissertação de Mestrado; Instituto militar de Engenharia, RJ, 2014.

2 KALPAKJIAN, S.; SCHMID, S. R.; Manufacturing Engineering and Technology; $7^{\text {a }}$ edição; Londres: Pearson, 2013.

3 LU, J.; Handbook of Measurements of Residual Stress, Vol. 2, Ed. SEM, 2a ed., pp. 417, 2005.

4 GUIMARÃES,L.R.; Estudo de Parâmetros Experimentais Envolvidos na Determinação de Macrotensões Residuais, em Tubos de Aço Inoxidável, pelo Método da Difração de Raios-X, Dissertação de Mestrado; Instituto de Pesquisas Energéticas e Nucleares Autarquia Associada à Universidade de São Paulo, SP, pp.92, 1990.

5 CULLITY, B.D..; STOCK, S. R. Elements of X-ray diffraction; $3^{\mathrm{a}}$ ed., Nova Jersey: Prentice Hall, 2001. 